\title{
artigo
}

\section{Perfil sociodemográfico e qualificação dos enfermeiros que atuam na assistência ao parto de rísco habitual}

\author{
Sociodemographic profile and qualification of nurses to low-risk childbirth care \\ Perfil sociodemográfico y cualificación profesional de los enfermeros en la atención del parto de bajo riesgo
}

\begin{abstract}
RESUMO
Objetivo: descrever o perfil sociodemográfico e formativo dos enfermeiros que atuam na assistência ao parto de risco habitual. Método: Estudo quantitativo, descritivo e exploratório. Foram aplicados questionários com enfermeiros que atuam na assistência ao parto de risco habitual. Os dados foram submetidos à análise estatística descritiva e apresentados através de frequência relativa, absoluta e média. Resultados: Os colaboradores são do sexo feminino, idade média de 39,6 anos, nortistas, em união estável, com um dependente financeiro. Média de 8,6 anos de estudo e 4,3 anos de trabalho na assistência. Graduados em instituições particulares, não possuem especialização na área de enfermagem obstétrica ou saúde da mulher, não realizaram atualização na área. A aprendizagem sobre a temática ocorreu durante a graduação e prática profissional. A rotatividade das escalas e a afinidade foram fatores relacionados ao trabalho de assistência ao parto. Conclusão: Aconselha-se atualização, capacitação e educação permanente.
\end{abstract}

DESCRITORES: Saúde materna; Saúde na fronteira; Equipe de Enfermagem; Enfermagem obstétrica.

\section{ABSTRACT}

Objective: to describe the sociodemographic profile and analyze to qualification of nurses to low-risk childbirth care. Method: This is a quantitative, descriptive, exploratory study with nurses who work in low-risk childbirth care, totaling six nurses who answered the sociodemographic questionnaire and the performance. Results: The employees are female, with an average age of 39.6 years, northerners, in a stable relationship, with a financial dependent. Average of 8.6 years of study and average of 4.3 years of care work. Graduation in private institutions. They have no specialization in the area of obstetric nursing or women's health. Learning about the theme occurred during graduation and professional practice. They did not update the area. Scale turnover and affinity were factors related to childbirth assistance. Conclusion: It is advisable to update, train and permanently educate nurses who work in childbirth care of usual risk.

DESCRIPTORS: Maternal health; Health border; Nursing team; Obstetric nursing.

\section{RESUMEN}

Objetivo: describir el perfil sociodemográfico y analizar la calificación de enfermeros que laboran en atención al parto por riesgo habitual. Método: Estudio cuantitativo, descriptivo y exploratorio. Se aplicaron cuestionarios a enfermeras que laboran en atención al parto de riesgo habitual, en un hospital de referencia en una ciudad fronteriza amazónica. Datos sometidos a análisis estadístico descriptivo y presentados mediante frecuencia relativa, absoluta y media. Resultados: Los empleados son mujeres, con una edad promedio de 39,6 años, norteños, en relación estable, con un dependiente económico. Promedio de 8,6 años de estudio y promedio de 4,3 años de trabajo asistencial. Graduación en instituciones privadas. No tienen especialización en el área de enfermería obstétrica o salud de la mujer. El aprendizaje sobre el tema ocurrió durante la graduación y la práctica profesional. No actualizaron el área. El cambio de escala y la afinidad fueron factores relacionados con la asistencia al parto. Conclusión: Es recomendable actualizar, formar y formar permanentemente a las enfermeras que laboran en la atención al parto de riesgo habitual.

DESCRIPTORES: Salud materna; Salud en la frontera; Equipo de enfermería; Enfermería obstétrica.

RECEBIDO EM: 13/01/2021 APROVADO EM: 18/02/2021

\section{Maria Perpétuo Socorro Lima Dantas}

Enfermeira. Especialista em Enfermagem Obstétrica pela Faculdade de Macapá, Macapá-AP.

ORCID: 0000-0002-2490-8836 


\section{Jordânia Vieira Silva}

Acadêmica de Enfermagem da Universidade Federal do Amapá. Macapá-AP, Brasil. Bolsista de Iniciação Científica. ORCID: 0000-0001-5308-1009

\section{Juliana Baia da Silva}

Enfermeira assistencial de Estratégia de Saúde da Família na Unidade Básica de Saúde José Alves Meireles. Coordenadora de Imunização do município de Tartarugalzinho - Amapá.

ORCID: 0000-0002-4111-7454

\section{Lise Maria Carvalho Mendes}

Enfermeira. Professora Assistente do curso de Enfermagem da Universidade Federal do Amapá. Doutoranda em Enfermagem em Saúde Pública pela Escola de Enfermagem de Ribeirão Preto - Universidade de São Paulo (EERP-USP), Ribeirão Preto-SP. ORCID: 0000-0001-9325-8382

\section{INTRODUÇÃO}

0 Brasil vivencia uma epidemia de intervenções desnecessárias na assistência obstétrica, que culminam em maiores taxas de cirurgias cesáreas sem indicação, infecção puerperal e mortalidade materna ${ }^{1}$. Sobre este aspecto, é importante destacar que a morte materna por causas evitáveis está intrinsicamente relacionada aos atrasos obstétricos, dentre os quais o terceiro tipo de atraso configura-se na demora da identificação dos sinais e sintomas de gravidade nas gestantes, parturientes e puérperas por parte dos profissionais de saúde ${ }^{2,3}$.

Nesta perspectiva, a enfermagem obstétrica relaciona-se a uma assistência ao parto baseada em evidências científicas, com adoção de intervenções em momentos oportunos para a manutenção da saúde materna e do recém-nascido ${ }^{4}$. Assim, para a redução da mortalidade materna verifica-se que é necessário a oferta de uma assistência obstétrica segura, em que os profissionais da saúde exerçam boas práticas de parto e nascimento e estejam em constante atualização profissional ${ }^{1,4-5}$.

No Brasil, a Rede Cegonha é um programa público de saúde responsável pela implementação de um novo modelo de atenção à saúde da mulher que propõe melhorias no atendimento às mulheres durantes a gestação, parto e puerpério, através de práticas de atenção à saúde baseada em evidências científicas ${ }^{6-7}$. Para tanto, fomenta a formação de enfermeiros obstetra, pois percebe a atuação desta categoria como estratégica no processo parturitivo, possibilitando assistência integral, respeitando o parto como um processo fisiológico e contribuindo para melhoria da saúde materna ${ }^{6-7}$.

Verifica-se que a região Norte brasileira, assim como outros países que se inserem nos contextos geográficos e socioculturais de fronteiras amazônicas ${ }^{8}$, possuem alguns entraves para assistência segura e pautada em conhecimentos científicos atualizados, a exemplo da escassez de profissionais qualificados e pouca ou nenhuma capacitação profissional para lidar com a diversidade linguística e cultural destas regiões?.

Desta forma, a seguinte questão norteadora foi formulada: qual o perfil formativo dos enfermeiros que atuam na assistência ao parto de risco habitual em regióes de fronteiras amazônicas? Assim, este estudo objetiva descrever o perfil sociodemográfico e formativo dos enfermeiros que atuam na assistência ao parto de risco habitual em um hospital referência da faixa de fronteira franco-brasileira. $\mathrm{O}$ conhecimento do perfil destes profissionais poderá ser utilizado como ferramenta e subsídio para direcionamento de atividades de educação continuada, corroborando para qualificação profissional da equipe e melhorando a assistência de saúde durante o trabalho de parto, parto e nascimento, bem como no gerenciamento de riscos à saúde, na avaliação da eficácia dos programas de treinamento e formação profissional.

\section{MÉTODO}

\section{Tipo de estudo}

Trata-se de estudo quantitativo, descritivo, exploratório, realizado em hospital refe- rência no atendimento a parto de risco habitual num município fronteiriço amazônico.

Especificou-se como critério de inclusão ser enfermeiro e já ter realizado assistência à mulher em trabalho de parto e parto de risco habitual. Elencou-se como critério de exclusão: estar afastado do serviço em decorrência de licença médica, de outra natureza ou férias.

A amostra foi do tipo censitária, ou seja, foram convidados todos enfermeiros que atuam no referido hospital, que contabilizam nove enfermeiros. Participaram da pesquisa seis destes. As ausências foram distribuídas em uma licença saúde, férias e uma recusa à participação. Agendou-se a coleta de dados previamente, em períodos vespertino e noturno, de acordo com a escala mensal de serviços, no período de setembro e outubro de 2017.

Destaca-se que os colaboradores responderam a um questionário estruturado contendo dados sociodemográficos e sobre o perfil formativo profissional para assistência ao parto. $\mathrm{O}$ questionário foi entregue aos participantes e devolvido à pesquisadora após preenchimento.

Norteou-se a apresentação e a análise dos dados pela estatística descritiva, em que foram definidas frequência relativa (\%), frequência absoluta (n) e média.

Os participantes assinaram Termo de Consentimento Livre e Esclarecido, atendendo à resolução 466, de dezembro de 2012, que aprova as diretrizes e normas regulamentadoras de pesquisas envolvendo seres humanos. Encaminhou-se o projeto ao Comitê de Ética da Universidade Federal do Amapá, que obteve aprovação com número de parecer 2.155.880. 
Tabela 01. Perfil sociodemográfico da população do estudo. Oiapoque, AmapáAP, Brasil. 2021

\section{$\mathbf{N}$}

Sexo

$\mathrm{F}$

$\mathrm{M}$

Faixa etária

20-29

30-49

Acima de 50 anos

Naturalidade

Norte

Nordeste

Sudeste

Estado civil

Solteiro(a)

Casado(a)/união estável

Viúvo (a)

Dependentes financeiros

Não possui

1

$2-3$

Fonte: Dados da pesquisa, 2021.

0

1

4

1

3

1

2

2

4

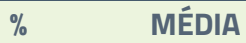

100

$16,7 \%$

$66,6 \%$

$16,7 \%$

$50 \%$

$16,7 \%$

$33,4 \%$

$33,4 \%$

$66,6 \%$

Tabela 2. Perfil profissional da população do estudo. Oiapoque, Amapá-AP,

Brasil. 2021.

Tempo de trabalho na assistência ao parto

Menos de 1 anos

$1-5$ anos

Mais de 5 anos

Instituição que realizou graduação

Pública

Privada

\section{Especialização Lato Sensu}

Não possui

Saúde coletiva

Gestão em saúde

Saúde da familia

Aprendizagem sobre obstetrícia

Graduação

Prática profissional

Cursos de atualização
$466,6 \%$

$233,4 \%$

$466,6 \%$

$233,4 \%$

$233,4 \%$

$1 \quad 16,7 \%$

$1 \quad 16,7 \%$

$233,4 \%$

$466,6 \%$

$233,4 \%$

\section{RESULTADOS}

Os colaboradores caracterizaram-se por majoritariamente serem do sexo feminino, com faixa etária média de 39,6 anos de idade, naturais da região Norte, casados ou com união estável e um dependente financeiro, dispostos na tabela 01 :

Apresentaram média de 4,3 anos de tempo de trabalho na assistência. A graduação dos colaboradores foi realizada em instituições particulares da região sudeste. Verifica-se também que possuem especialização Lato Sensu, mas não na área de enfermagem obstétrica ou saúde da mulher. A aprendizagem sobre obstetrícia ocorreu majoritária durante o curso de graduação e a prática profissional.

Predominaram aqueles que não realizaram atualização na área de saúde sexual e reprodutiva. Quando questionados porque atuam na área de saúde da mulher, verificou-se que a rotatividade das escalas e a afinidade com a área foram os principais $\mathrm{fa}$ tores mencionados. O desejo por mudar de área de atuação foi verbalizado por alguns, mediante as justificativas de sobrecarga de trabalho e de desvalorização profissional. Como podemos verificar na tabela 2:

\section{DISCUSSÃO}

A equipe de enfermagem que atua na assistência ao trabalho de parto, parto e nascimento na fronteira amazônica é predominantemente feminina. Estes dados coadunam com o perfil dos enfermeiros brasileiros, em que as mulheres correspondem a $84,6 \%$ da força de trabalho ${ }^{10}$. Verifica-se sobre este aspecto, que, historicamente, a responsabilidade pelo trabalho vinculado às relações de cuidado em geral, tanto de forma remunerada e profissionalizante, como também o trabalho não remunerado na esfera privada, é atribuído de forma desigual e prioritária às mulheres ${ }^{11}$.

Essas bases ideológicas naturalizam um processo de desvalorização das profissões relacionadas ao cuidado, em que se observa uma menor remuneração, condições precárias de trabalho, turnos exaustivos, sobreposição de contratos de trabalho, escassez ou inadequação de equipamentos de proteção individual (EPIs) ${ }^{11-12}$. Neste sentido, estudo 


\section{Cursos de atualização profissional na área de saúde sexual e reprodutiva}

$\begin{array}{lcc}\text { Não realizaram } & 4 & 66,6 \% \\ \text { Realizaram há mais de } 2 \text { anos } & 2 & 33,4 \% \\ \begin{array}{l}\text { Realizaram há menos de } 2 \text { anos } \\ \text { Motivação para trabalhar na área de saúde da mulher }\end{array} & 0 & - \\ \text { Rotatividade das escalas } & 3 & 50 \% \\ \text { Afinidade com a área } & 3 & 50 \% \\ \text { Motivações ao desejo de modificar a área de atuação profissional } & \\ \text { Sobrecarga de trabalho } & 6 & 100 \% \\ \text { Desvaloriozação Profissional } & 5 & 72,7 \% \\ \text { Fonte: Dados da pesquisa, 2021. } & \end{array}$

comparando os processos de saúde e doença entre enfermeiros e enfermeiras observou que as enfermeiras expressaram maiores níveis de desgaste do que os homens, mediante experiências que destacam a presença de cansaço e sobrecarga de trabalho, relacionados às duplas jornadas de trabalho, conexas aos cuidados com filhos e outros familiares ${ }^{13-14}$.

Neste estudo, verifica-se ainda que a verbalização sobre a insatisfação com a jornada de trabalho e desvalorização sobre o trabalho esteve presente. Ressalta-se sobre este aspecto, que, para ofertar uma assistência de qualidade, é necessário que haja compreensão acerca do esgotamento profissional, que se torna um impeditivo para uma assistência segura. Assim, nota-se a necessidade de políticas públicas que promovam salários e jornadas de trabalhado dignos.

No tocante à naturalidade, destaca-se que a mão de obra atuante na assistência ao parto na fronteira em questão é composta predominantemente por migrantes de outros estados brasileiros, com ênfase às regiões norte e nordeste. Este aspecto é observado em outras categorias profissionais, em que há mão-de-obra oriunda de outras localidades $^{8,15-16}$. Quanto ao estado marital, verifica-se que a presença de um (a) companheiro (a) pode oferecer conforto, num cenário em que os profissionais estão distantes de sua cidade natal, pois são majoritariamente naturais de lugares distintos aos que desenvolvem suas atividades laborais. Estudo recente observou que o estado conjugal influenciou os domínios psicológico, nível de independência, relaçôes sociais e ambiente, em que os indiví- duos que possuem companheiro(a) apresentaram melhores escores de qualidade de vida quando comparados aos que não possuíam ${ }^{17}$.

Observa-se que a média de anos estudos é menor quando comparada a enfermeiros que atuam na assistência ao parto em grandes centros urbanos ${ }^{10}$. Apesar dos colaboradores possuírem certificação de pós-graduação Lato Sensu, estas não conferem capacitação para atuação no campo de enfermagem obstétrica e assistência à saúde da mulher, parto e nascimento. Este perfil foi observado em outros estados da região Norte, em enfermeiros que atuam na assistência ao parto ${ }^{18}$.

A ausência de especialização na área obstétrica é referenciada na literatura como um impeditivo para assistência segura, pois além de não assegurar habilitação técnica, a ausência de capacitação específica acarreta a diminuição da autonomia da equipe de enfermagem $^{8,19}$. Além do fator elencado, a formação em enfermagem obstétrica está associada a um número reduzido de intervenções desnecessárias durante o trabalho de parto e parto $^{19}$, ao emprego das boas práticas de parto e nascimento e ao incentivo ao aleitamento materno na primeira hora de vida ${ }^{20}$.

O déficit formativo e a ausência de capacitação do enfermeiro obstetra é apontando na literatura como um dos fatores associados à dificuldade do exercício das boas práticas de parto e nascimento preconizadas pela Organização Mundial de Saúde. A literatura também infere a dicotomia entre a prática profissional e a legislação, além da resistência dos profissionais da categoria médica frente à atuação da enfermagem obstétrica $^{21}$. Verifica-se ainda que a educação permanente não ocorre de maneira rotineira, o que dificulta o desenvolvimento da atitude colaborativa entre a equipe ${ }^{21}$.

A presença de enfermeiros obstétricos nas instituições hospitalares mostra-se capaz de reconfigurar o modelo assistencial, contribuindo com mudanças por meio de uma atuação autônoma, colaborativa e de qualidade em atendimento às políticas públicas de saúde nacionais e internacionais relacionadas ao trabalho de parto, parto e nascimento ${ }^{4,23}$. Sobre este aspecto, é visto a necessidade de oferta de educação permanente por parte da gestão pública aos profissionais atuantes e aos que são recém-chegados, com a finalidade de fortalecer a prática profissional, pautando a assistência de enfermagem às famílias que vivenciam o parto de risco habitual nas melhores evidências científicas.

\section{CONCLUSÃO}

O estudo concluiu que o perfil formativo dos enfermeiros que atuam na assistência ao parto de risco habitual em um hospital referência da faixa de fronteira franco-brasileira ensejam atualização, capacitação e educação permanente nesta área assistencial. Esta investigação apresenta resultados preliminares sobre o perfil profissional dos enfermeiros atuantes na assistência ao trabalho de parto, parto e nascimento em áreas de fronteiras amazônicas, corroborando às futuras investigações sobre o ensino profissional especializado e suas interfaces com a realidade da assistência obstétrica prestada à sociedade.

O conhecimento acerca do perfil e das competências formativas destes profissionais poderá fornecer subsídios para direcionamento de atividades de capacitação, proporcionando maior qualificação profissional e, por conseguinte, melhora da assistência ao trabalho de parto e parto. Foram consideradas limitações do estudo a recusa de três enfermeiros pelos seguintes motivos: férias, licença saúde e recusa de participação, o que impossibilita a generalização dos achados. O estudo apresentado é um exemplo não somente $\mathrm{da}$ realidade específica analisada, mas se insere como protótipo de um contexto mais amplificado, vivenciado em áreas remotas. . 


\section{REFERÊNCIAS}

1. Gomes SC, Teodoro LPP, Pinto AGA, Oliveira DR de, Quirino G da S, Pinheiro AKB. Rebirth of childbirth: reflections on medicalization of the Brazilian obstetric care. Rev. bras. enferm [Internet]. 2018 [cited 2021 jan 2]; 71(5). Available from: http://www.scielo.br/pdf/ reben/v71n5/0034-7167- reben-71-05-2594.pdf

2. Pacagnella RC, Nakamura-Pereira Ma, Gomes-Sponholz F, Aguiar RALP de, Guerra GVQL, Diniz CSG et al . Maternal Mortality in Brazil: Proposals and Strategies for its Reduction. Rev. Bras. Ginecol. Obstet[internet]. 2018[cited 2021 jan 2];40(9):501-506. Doi: 10.1055/s-0038-1672181.

3. Silva JMP, Fonseca SC, Dias MAB, Izzo AS, Teixeira GP, Belfort PP. Conceitos, prevalência e características da morbidade materna grave, near miss, no Brasil: revisão sistemática. Rev. Bras. Saude Mater. Infant[internet]. 2018[cited 2021 jan 2]; 18(1): 7-35. Doi: 10.1590/1806-93042018000100002.

4. Alvares AS, Corrêa AC de P, Nakagawa JTT, Teixeira RC, Nicolini AB, Medeiros RMK. Práticas humanizadas da enfermeira obstétrica: contribuições no bem-estar materno. Rev. bras. enferm [Internet]. 2018 [cited 2021 jan 2]; 71(6). Doi: 10.1590/00347167-2017-0290

5. Oliveira APC de, Gabriel M, Poz MRD, Dussault G. Challenges for ensuring availability and accessibility to health care services under Brazil's Unified Health System (SUS). Ciênc. saúde coletiva. [Internet]. 2017 [cited 2021 jan 2]; 22(4). Doi: 10.1590/141381232017224.31382016

6. BRASIL. Ministério da Saúde. Diretrizes nacionais de assistência ao parto normal: versão resumida [internet]. 2017.

7. Oliveira EM, Celento DD. A temática da Rede Cegonha e a inserção do enfermeiro nesse contexto. Revista de Saúde. 2016; 07 (1): 33-38.

8. Fernandes KG, Souza RT, Leal MC, Moura EC, Santos LM, Cecatti JG. Ethnic differences in maternal near miss. Arch Gynecol Obstet[internet]. 2017[cited 2021 jan 2];29(6):1063-1070. Doi:10.1007/s00404-017-4530-6

9. Silva AC, Mendes LMC, Monteiro RS, Silva RA, Pinheiro AKB. Birth and childbirth on the French-Brazilian border: nurses' perceptions. Cogitare enferm[internet]. 2020[cited 2021 jan 2]; 25. Doi:10.5380/ce.v25i0.67820

10. Machado MH, Mônica W, Vieria M, Oliveira E, Lemos W , Aguiar Filho $W$ et al. Aspectos gerais da formação da enfermagem: o perfil da formação dos enfermeiros, técnicos e auxiliares. Enferm. Foco[internet] 2016[cited 2021 jan 2]; 6(2):15-34. Available from: https://docs.bvsalud.org/biblioref/2018/06/881147/687-17451-sm.PDF

11. Lopes MJM, Leal SMC. A feminização persistente na qualificação profissional da enfermagem brasileira. Cad Pagu. 2005; 24:105-125.

12. Pereira AV, Oliveira SSR. Migração de demandas entre as esferas público-privadas sob a ótica das relações de gênero: um estudo com enfermeiras e enfermeiros. Interface - Comunicação, Saúde, Educação [interface]. 2019[cited 2021 jan 3]; 23 :e170448. Doi: 10.1590/Interface. 170448
13. Pereira AV. O cotidiano de enfermeiras e enfermeiros: relações de gênero, a partir do tempo no hospital. Rev. Latino-Am. Enfermagem [Internet]. 2015[cited 2021 jan 3]; 23(5):945-953. Doi: 10.1590/0104-1169.0485.2635

14. Rodrigues BC, Lima MF, Maschio NB, Oliveira GL, Corrêa ACP, Higarashi IH. Being a mother and a nurse: issues about gender and overlapping social roles. Rev Rene[internet]. 2017[cited 2021 jan 3];18(1):91. Doi:10.15523/2175-6783.2017000100013

15. Mendes LMC, Silva Neto AS, Andrade RF. Health care of brazilian women working in clandestine mining sites with in the French Amazon rain forest. International Journal of Development Research [Internet]. 2019[cited 2021 jan 3];1(3):1-4. Available from: http://www.journalijdr.com/health-care-brazilian-women-working-clandestine-mining-sites-within-french-amazon-rainforest-0

16. Sousa LP de, Monteiro RS, Nascimento VB, Silva Neto AS da, Mendes LMC. Performance of the nursing team in the rapid HIV test. J Nurs UFPE on line[internet]. 2020[cited 2021 jan 3];14:e244420 Doi: 10.5205/1981-8963.2020.244420

17. Fernandes JS, Miranzi SSC, Iwamoto HH, Tavares DMS, Santos CB. Qualidade de vida dos enfermeiros das equipes de saúde da família: a relação das variáveis sociodemográficas. Texto contexto - enferm. [Internet]. 2010[cited 2021 jan 3]; 19(3):434-442. Doi: 10.1590/S0104-07072010000300004

18. Ribeiro JK, Santana MDO, Sá JS, Silva MA. The challenges of humanization in nursing care during normal birth in a municipal hospital in the state of tocantins. Revista Multidebates[internet]. 2019[cited 2021 jan 3]; 3(1). Available from: http://revista.faculdadeitop.edu.br/index.php/revista/article/view/141/147

19. Melo AA, Diaz CMG, Zamberlan C, Antunes B, Marques CT, Silveira GB, Pinheiro BF, Kruel CS. Childbirth care profile in a usual risk maternity hospital: type of delivery and interventions. Research, Society and Development[internet]. 2020[cited 2021 jan 3]; 9(2). Doi: 10.33448/rsd-v9i2.1905.

20. Carrion KT, Souza LC, Marques CT, Neves ET, Costenaro RG, Marchinski AM et al. Repercussions of neonatal good practices from the children's assistance profile. Research, Society and Development[internet]. 2020[cited 2021 jan 3]; 9(6): e122963451. Doi: 10.33448/rsd-v9i6.3451.

21. Magalhães TTS, Taffner VBM. Dificuldades para a atuação autônoma do enfermeiro obstetra no Brasil. REVISA[internet]. 2020[cited 2021 jan 3];9(4):685-97. Doi: 10.36239/revisa.v9.n4. p685a697

22. Amaral RCS, Alves VH, Pereira AV, Rodrigues DP, Silva LA, Marchiori GRS. Obstáculos a prática da enfermeira obstétrica. Esc Anna Nery[internet] 2019[cited 2021 jan 3];23(1):e20180218 Doi : 10.1590/2177-9465-EAN-2018-0218

23. Amaral RCS, Alves VH, Pereira AV, Rodrigues DP, Silva LA, Marchiori GRS. A inserção da enfermeira obstétrica no parto e nascimento: obstáculos em um hospital de ensino no Rio de Janeiro. Esc. Anna Nery [Internet]. 2019 [cited 2021 Jan 08]; 23(1):e20180218. Doi: 10.1590/2177-9465-ean-2018-0218. 